\title{
REMARKS ON SPACES WITH SPECIAL TYPE OF $k$-NETWORKS
}

\author{
By \\ Masami SAKaI
}

\begin{abstract}
We negatively answer the following questions posed by Y. Ikeda and Y. Tanaka. (1) Does every closed image of a space $X$ with a star-countable $k$-network have a star-countable $k$-network, or a point-countable $k$-network? (2) Is every space $X$ with a locally countable $k$-network a $\sigma$-space, or a space in which every closed subset is a $G_{\sigma}$-set?
\end{abstract}

\section{Introduction}

All spaces we consider here are completely regular Hausdorff and all maps are continuous and onto. A collection of subsets of a space is said to be star-countable (resp. point-countable) if each element (resp. single point) meets only countably many members. Obviously a star-countable collection is pointcountable. A collection $\mathscr{P}$ of subsets of a space $X$ is called a $k$-network if whenever $K$ is a compact subset of an open set $U$, there exists a finite subset $\mathscr{P}^{\prime}$ of $\mathscr{P}$ such that $K \subset \bigcup \mathscr{P}^{\prime} \subset U$. If we replace "compact" by "single point", then $\mathscr{P}$ is called a network. A space with a $\sigma$-locally finite network is called a $\sigma$-space.

Concerning spaces with special type of $k$-networks, Y. Ikeda and Y. Tanaka posed the following questions in [7], see also [10] and [12].

QUESTIONS. (1) Does every closed image of a space $X$ with a star-countable $k$-network have a star-countable $k$-network, or a point-countable $k$-network?

(2) Is every space $X$ with a locally countable $k$-network a $\sigma$-space, or a space in which every closed subset is a $G_{\sigma}$-set?

The question (1) has a positive answer under some conditions.

Received July 31, 1995.

Revised November 20, 1995. 
THEOREM 1 [10]. Let $f: X \rightarrow Y$ be a closed map such that $X$ has a pointcountable $k$-network. If one of the following properties holds, then $Y$ has a point-countable $k$-network.

(a) $X$ is a $k$-space,

(b) Each point of $X$ is a $G_{\sigma}$-set,

(c) $X$ is a normal, isocompact space,

(d) For each $y \in Y \partial f^{-1}(y)$ is Lindelöf, where $\partial f^{-1}(y)$ is the boundary of $f^{-1}(y)$.

The question (2) has a positive answer if $X$ is a $k$-space, in fact a $k$-space with a locally countable $k$-network is the topological sum of $\aleph_{0}$-spaces, see [7] (cf. [9]).

In this paper we give counterexamples for the questions and slightly generalize the case (d) of Theorem 1 .

\section{Counterexamples}

In this paper we endow $\omega_{1}$ with the discrete topology. For a subset $A$ of a discrete space $D$ we put $A^{*}=C l_{\beta D} A-A$, where $\beta D$ is the Stone-Čech compactification of $D$.

For convenience, we call a space $X$ a $C F$-space if every compact subset of $X$ is finite. If $X$ is a CF-space, then the collection $\{\{x\}: x \in X\}$ is obviously a start-countable $k$-network of $X$.

Recall that the one-point compactification of $\omega_{1}$ does not have any pointcountable $k$-network. In fact, a compact space with a point-countable $k$-network is metrizable, see Theorem 3.1 in [3].

Hence the following example shows that the first question has a negative answer.

Example 1. There exists a closed map $f$ from a CF-space $X$ onto the onepoint compactification of $\omega_{1}$.

Proof. A point $z$ of a space $Z$ is called a weak P-point if $z \notin \bar{E}$ for any countable $E \subset Z-\{z\}$. It is known that $\omega^{*}$ contains weak P-points [8]. Hence we can see that the set $P=\left\{p \in \omega_{1}^{*}: p\right.$ is a weak P-point in $\left.\omega_{1}^{*}\right\}$ is dense in $\omega_{1}^{*}$.

We set $X=\omega_{1} \cup P$, the subspace of $\beta \omega_{1}$. It is easy to check that $X$ is a CFspace, because a compact space in which every point is a weak P-point is finite, and every convergent sequence of $\beta \omega_{1}$ is finite. Let $Y$ be the space obtained by 
collapsing the closed set $P$ to one point, and let $f$ the canonical map from $X$ onto $Y$. Then $f$ is a closed map, and since the closure of an infinite subset of $\omega_{1}$ intersects with $\mathrm{P}, Y$ is the one-point compactification of $\omega_{1}$.

A collection $\mathscr{P}$ of subsets of a space $X$ is called a cs-network if whenever $\sigma$ is a sequence converging to a point $x$ such that $\sigma \cup\{x\} \subset U$ with $U$ open in $X$, then there exists a $P \in \mathscr{P}$ such that $x \in P \subset U$ and $\sigma$ is eventually in $P$.

It is known that every space is the perfect image of an extremally disconnected space, see [13], where a space is extremally disconnected if the closure of an open set is open. Since every convergent sequence of an extremally disconnected space is finite, every space is the perfect image of a space with a point-countable cs-network. So, it is natural to ask whether every space is the closed image of a space with a point-countable $k$-network.

The author does not know if it is true. But, at least, the following holds.

Proposition. Every space is the quotient image of a CF-space.

Proof. Let $Z$ be a space. As noted above, $Z$ is the perfect image of an extremally disconnected space $Y$. For each point $y \in Y$, let $Y_{y}$ be the space obtained by isolating all points of $Y$ but $y$. Then $Y$ is canonically the quotient image of the topological sum $X=\bigoplus\left\{Y_{y}: y \in Y\right\}$. If $K$ is an infinite compact subset of $Y_{y}$, then it contains a non-trivial convergent sequence to $y$. Hence $Y$ must have a non-trivial convergent sequence. This is a contradiction. Thus $X$ is a CF-space, and $Z$ is the quotient image of $X$.

If $X$ is a locally countable CF-space, then the collection $\{\{x\}: x \in X\}$ is obviously a locally countable $k$-network of $X$.

A space is countably metacompact if every countable open cover has a point-finite open refinement. It is not difficult to check that a space $X$ is countable metacompact iff whenever $\left\{C_{n}\right\}$ is a decreasing sequence of closed sets of $X$ with empty intersection, there exist open sets $U_{n} \supset C_{n}$ with $\bigcap\left\{U_{n}: n \in \omega\right\}=\varnothing$.

Recall the diagram below:

$\sigma$-space $\rightarrow$ perfect (every closed set is a $G_{\sigma}$-set) $\rightarrow$ countably metacompact

Hence the following example shows that the second question is also negative. 
EXAMPLE 2. There exists a locally countable CF-space $X$ which is not countably metacompact.

Proof. Let $D$ be a set of cardinality $2^{\omega}$. Let $\left\{P_{\alpha}: \alpha<2^{\omega}\right\}$ be an almost disjoint family of countable infinite subsets of $D$ such that for every uncountable $P \subset D$ there exists some $\alpha$ with $P_{\alpha} \subset P$. Such a family exists, for example see [1, Example 4.2]. For each $\alpha$, let $\left\{P_{\alpha n}: n \in \omega\right\}$ be a disjoint family of infinite subsets of $P_{\alpha}$. We set $\mathscr{P}=\left\{P_{\alpha n}: \alpha<2^{\omega}, n \in \omega\right\}$. We endow $D$ with the discrete topology. For each $\alpha, n$, pick a point $p_{\alpha n} \in P_{\alpha n}^{*}$.

We set $X=D \cup\left\{p_{\alpha n}: \alpha<2^{\omega}, n \in \omega\right\}$, the subspace of $\beta D$. Since $\mathscr{P}$ is almost disjoint, $X-D$ is a closed discrete subset of $X$. $X$ is obviously a locally countable CF-space.

We see that $X$ is not countably metacompact. For each $n \in \omega$, let $C_{n}=\left\{p_{\alpha k}: \alpha<2^{\omega}, k \geq n\right\}$. Each $C_{n}$ is closed in $X$ and $\bigcap\left\{C_{n}: n \in \omega\right\}=\varnothing$. Assume that there exist open sets $U_{n} \supset C_{n}$ with $\bigcap\left\{U_{n}: n \in \omega\right\}=\varnothing$. Since $D$ is uncountable, there exists $n \in \omega$ such that $D-U_{n}$ is uncountable. Then there exists some $\alpha$ with $P_{\alpha} \subset D-U_{n}$. Hence the closure of $D-U_{n}$ must contain $p_{\alpha n} \in C_{n}$. This is a contradiction. Thus $X$ is not countably metacompact.

\section{A generalization}

In this section we slightly generalize the case $(d)$ in Theorem 1.

A subset $S$ of a space $X$ is z-embedded in $X$ if every zero-set of $S$ is the restriction to $S$ of some zero-set of $X$. A map $f: X \rightarrow Y$ is compact-covering if every compact subset of $Y$ is the image of a compact subset of $X$. For realcompact spaces, see [5].

Lemma 1. Let $f: X \rightarrow Y$ be a closed map. Then (1) and (2) below hold.

(1) If $Y$ is realcompact and for each $y \in Y f^{-1}(y)$ is realcompact, $z-$ embedded in $X$, then $X$ is realcompact. [2, Theorem 3.9]

(2) If $X$ is realcompact, then $f$ is compact-covering. [4, Theorem 3.4]

Corollary. Let $f: X \rightarrow Y$ be a closed map. If for each $y \in Y \partial f^{-1}(y)$ is realcompact, $z$-embedded in $X$, then $f$ is compact-covering.

Proof. Let $K$ be a compact subset of $Y$. For each $y \in K$, choose any $x_{y} \in f^{-1}(y)$. We set:

$$
A_{y}= \begin{cases}\partial f^{-1}(y) & \text { if } \partial f^{-1}(y) \neq \varnothing \\ \left\{x_{y}\right\} & \text { if } \partial f^{-1}(y)=\varnothing\end{cases}
$$


Then the set $A=\cup\left\{A_{y}: y \in K\right\}$ is closed in $X$, hence the restricted map $g=f \mid A: A \rightarrow K$ is a closed map. By lemma $1(1), A$ is realcompact. By Lemma 1 (2), $g$ is compact-covering. So there exists a compact set $K^{\prime} \subset A$ with $f\left(K^{\prime}\right)=K$.

Let $\mathscr{P}$ be a collection of subsets of a space $X, \mathscr{P}$ is called a wcs*-network of $X$ if whenever $\left\{x_{n}\right\}$ is a sequence converging to a point $x \in X$ and $U$ is an open set of $X$ with $\{x\} \cup\left\{x_{n}\right\} \subset U$, there exists a $P \in \mathscr{P}$ such that $P \subset U$ and $P$ contains some subsequence of $\left\{x_{n}\right\}$.

Lemma 2 [11, Proposition 1.2.(1)]. Let $\mathscr{P}$ be a point-countable cover of $X$. Then $\mathscr{P}$ is a $k$-network of $X$ iff $\mathscr{P}$ is a wcs ${ }^{*}$-network of $X$ and each compact subset of $X$ is sequentially compact.

A Lindelöf space is realcompact [5, 8.2], and every Lindelöf subspace of a space $X$ is z-embedded in $X[6,5.3]$. Hence the following theorem generalizes the case $(d)$ of Theorem 1 .

TheOREM 2. Let $f: X \rightarrow Y$ be a closed map such that for each $y \in Y$ $\partial f^{-1}(y)$ is realcompact, $z$-embedded in $X$. If $X$ has a point-countable $k$-network, then so does $Y$.

Proof. The idea of the proof is due to [10].

Let $K$ be a compact subset of $Y$. By the corollary above, there exists a compact set $K^{\prime}$ of $X$ with $f\left(K^{\prime}\right)=K$. As noted in the second section, a compact space with a point-countable $k$-network is metrizable, so $K^{\prime}$ is metrizable. Therefore $K$ is metrizable, in particular sequentially compact.

By Lemma 2 we have only to construct a point-countable wcs*-network of $Y$.

Let $\mathscr{P}$ be a point-countable $k$-network of $X$. For each $y \in Y$ choose any $x_{y} \in f^{-1}(y)$. We set $A=\left\{x_{y}: y \in Y\right\}$ and $\mathscr{P}^{\prime}=\{f(P \cap A): P \in \mathscr{P}\}$. Obviously $\mathscr{P}^{\prime}$ is point-countable. We see that $\mathscr{P}^{\prime}$ is a wcs*-network of $Y$. Let $\left\{y_{n}: n \in \omega\right\}$ be a sequence converging to a point $y \in Y$, and $U$ be an open set of $Y$ with $K \subset U$, where $K=\{y\} \cup\left\{y_{n}: n \in \omega\right\}$. Since the set $J=\partial f^{-1}(y) \cup\left\{x_{n}: n \in \omega\right\}$, where $x_{n}=x_{y_{n}}$, is closed in $X$, the restricted map $g=f \mid J: J \rightarrow K$ is a closed map. By Lemma $1(1), J$ is realcompact. By Lemma $1(2), g$ is compact-covering. Hence there exists a compact set $J^{\prime} \subset J$ such that $g\left(J^{\prime}\right)=K$. Note that $\left\{x_{n}: n \in \omega\right\} \subset$ $J^{\prime} \subset f^{-1}(U)$. Since $\mathscr{P}$ is a $k$-network of $X$, there exists a $P \in \mathscr{P}$ such that 
$P \subset f^{-1}(U)$ and $P \cap\left\{x_{n}: n \in \omega\right\}$ is infinite. The set $f(P \cap A)$ is a desired one. Thus $\mathscr{P}^{\prime}$ is a wcs*-network of $Y$.

Lemma 1 (2) and the same idea as the proof of Theorem 2 lead to the following theorem.

TheOREM 3. Let $f: X \rightarrow Y$ be a closed map such that $X$ is realcompact. If $X$ has a point-countable $k$-network, then so does $Y$.

\title{
References
}

[1] B. Balcar and P. Simon, Disjoint refinement, in: Handbook of Boolean Algebras, NorthHolland, 1989, vol. 2, 332-386.

[ 2 ] R. L. Blair, Filter characterizations of $z$-, $C^{*}$-, and $C$-embeddings, Fund. Math., 90 (1976), 285300.

[ 3 ] D. Burke and E. Michael, On certain point-countable covers, Pacific J. Math., 64 (1976), 79-92.

[4] N. Dykes, Mappings and realcompact spaces, Pacific J. Math., 31 (1969), 347-358.

[5] L. Gillman and M. Jerison, Rings of continuous functions, Van Nostrand, Princeton, 1960.

[6] M. Henriksen and D. G. Johnson, On the structure of a class of archimedean lattice-ordered algebras, Fund. Math., 50 (1961), 73-94.

[ 7 ] Y. Ikeda and Y. Tanaka, Spaces having star-countable $k$-networks, Topology Proc., 18 (1993), 107-132.

[ 8 ] K. Kunen, Weak P-points in $N^{*}$, Coll. Math. Soc. János Bolyai, 23 (1978), 741-749.

[9] S. Lin, Spaces with a locally countable $k$-network, Northeastern Math. J., 6 (1990), 39-44.

[10] S. Lin and Y. Tanaka, Point-countable $k$-networks, closed maps, and related results, Topology and Appl., 59 (1994), 79-86.

[11] Y. Tanaka, Point-countable covers and $k$-networks, Topology Proc., 12 (1987), 327-349.

[12] Y. Tanaka, Theory of $k$-networks, Q \& A in General Topology, 12 (1994), 139-164.

[13] R. G. Woods, A survey of absolutes of topological spaces, Topological Structures II, Math. Centre Tracts, 116 (1979), 323-362.

\author{
Department of Mathematics \\ Kanagawa University \\ Yokohama, 221, Japan \\ e-mail: msakai@kamome.cc.kanagawa-u.ac.jp
}

